\title{
LOCAL SHARP MAXIMAL FUNCTIONS, GEOMETRICAL MAXIMAL FUNCTIONS AND ROUGH MAXIMAL FUNCTIONS ON LOCAL MORREY SPACES WITH VARIABLE EXPONENTS
}

\author{
Tat-Leung Yee, Ka Luen Cheung, KwoK-Pun Ho and Chun Kit Suen
}

Abstract. We study the local Morrey spaces with variable exponents. We show that the local block space with variable exponents are pre-duals of the local Morrey spaces with variable exponents. Using this duality, we establish the extrapolation theory for the local Morrey spaces with variable exponents. The extrapolation theory gives the mapping properties for the local sharp maximal functions, the geometric maximal functions and the rough maximal function on the local Morrey spaces with variable exponents.

Mathematics subject classification (2010): 42B20, 42B35, 46E30.

Keywords and phrases: Variable Lebesgue space, Local Morrey space, local block space, extrapolation, local sharp maximal function, geometric maximal function, rough maximal function.

\section{REFERENCES}

[1] O. Blasco, A. Ruiz And L. Vega, Non Interpolation in Morrey-Campanato and Block Spaces, Ann. Sc. Norm. Super. Pisa, Cl. Sci., Ser. 4, 28 (1999), 31-40.

[2] V. I. Burenkov AND H. V. Guliyev, Necessary and sufficient conditions for boundedness of the maximal operator in local Morrey-type spaces, Stud. Math. 163 (2004), 157-176.

[3] V. I. Burenkov, H. V. Guliyev and V. S. Guliyev, Necessary and sufficient conditions for boundedness of the fractional maximal operator in the local Morrey-type spaces, J. Comp. Appl. Math. 208 (2007), 280-301 (2007).

[4] V. I. Burenkov, V. S. Guliyev, T. V. Tarary kova and A. Serbetci, Necessary and sufficient conditions for the boundedness of genuine singular integral operators in Local Morrey-type spaces, Dokl. Akad. Nauk 422 (2008), 11-14.

[5] V. I. BURENKov AND V. S. GuliYev, Necessary and sufficient conditions for the boundedness of the Riesz potential in local Morrey-type spaces, Potential Anal. 30 (2009), 211-249.

[6] V. Burenkov, A. Gogatishvili, V. S. Guliyev and R. Mustafayev, Boundedness of the fractional maximal operator in local Morrey-type spaces, Compl. Variabl. Ellipt. Equat. 55 (2010), 739-758.

[7] V. Burenkov and E. Nursultanov, Description of interpolation spaces for local Morrey-type spaces, Proc. Steklov Inst. Math. 269 (2010), 46-56.

[8] V. Burenkov, A. Gogatishvili, V. S. Guliyev and R. Mustafayev, Boundedness of the Riesz potential in local Morrey-type spaces, Potential Anal. 35 (2011), 67-87.

[9] K. L. CHEUNG AND K.-P. Ho, Boundedness of Hardy-Littlewood maximal operator on block spaces with variable exponent, Czechoslovak Math. J., 64 (2014), 159-171.

[10] D. Cruz-Uribe, A. Fiorenza And C. Neugebauer, The maximal function on variable $L^{p}$ spaces, Ann. Acad. Sci. Fenn. Math. 28 (2003), 223-238.

[11] D. Cruz-Uribe, J. Martell and C. PÉrez, Weights, Extrapolation and the Theory of Rubio de Francia, O perator Theory: Advance and Applications, 215, Birkhäuser Basel, 2011.

[12] D. CruZ-Uribe And A. FiorenzA, Variable Lebesgue Spaces, Birkhäuser, 2013.

[13] L. Diening, Maximal functions on generalized Lebesgue spaces $L^{p(\cdot)}$, Math. Inequal. Appl. 7 (2004), 245-253. 
[14] L. Diening, P. Harjulehto, P. Hästö and M. RuŽičKa, Lebesgue and Sobolev Spaces with Variable Exponents, Springer, 2011.

[15] C. Fefferman and E. Stein, $H^{p}$ spaces in several variables, Acta Math. 129 (1972), 137-193.

[16] A. Gogatishvili and R. Mustafayev, Dual spaces of local Morrey-type spaces, Czech. Math. J. 61 (2011) 609-622.

[17] V. S. GuLIYEV, Generalized local Morrey spaces and fractional integral operators with rough kernel, J. Math. Sci. 193 (2013), 211-227.

[18] V. S. GuliYeV, J. HASANOV AND S. SAmKo, Maximal, potential and singular operators in the local "complementary" variable exponent Morrey type spaces, J. Math. Anal. Appl. 401 (2013), 72-84.

[19] K.-P. Ho, Vector-valued singular integral operators on Morrey type spaces and variable TriebelLizorkin-Morrey spaces, Ann. Acad. Sci. Fenn. Math. 37 (2012), 375-406.

[20] K.-P. Ho, Extrapolation, John-Nirenberg inequalities and characterizations of BMO in terms of Morrey type spaces, Rev. Mat. Complut. 30 (2017), 487-505.

[21] K.-P. Ho, Mixed norm Lebesgue spaces with variable exponents and applications, Riv. Mat. Univ. Parma. 9 (2018), 21-44.

[22] K.-P. Ho, Singular integral operators with rough kernel on Morrey type spaces, Studia Math., 244 (2019), 217-243.

[23] K.-P. Ho, Sublinear operators on weighted Hardy spaces with variable exponents, Forum Math. 31, (2019), 607-617.

[24] K.-P. Ho, Definability of singular integral operators on Morrey-Banach spaces, J. Math. Soc. Japan 72 (2020), 155-170.

[25] K.-P. Ho, Extrapolation to Herz spaces with variable exponents and applications, Rev. Mat. Complut. (2019). (published online) DOI: https://doi .org/10.1007/s13163-019-00320-3.

[26] K.-P. Ho, Spherical maximal function, maximal Bochner-Riesz mean and geometrical maximal function on Herz spaces with variable exponents, Rend. Circ. Mat. Palermo, II. Ser (2020). https://doi.org/10.1007/s12215-020-00511-8

[27] K.-P. Ho, Sublinear operators on block type space, Science China Math. (2020) 63, 1107-1124.

[28] K.-P. Ho, Stein-Weiss inequalities for radial local Morrey spaces, Port. Math. (2019) 76, 301-310.

[29] K.-P. Ho, Sublinear operators on mixed norm Hardy spaces with variable exponents, Atti Accad. Naz. Lincei Rend. Lincei Mat. Appl. (to appear).

[30] K.-P. Ho, Boundedness of operators and inequalities on Morrey-Banach spaces, Publ. Res. Inst. Math. Sci. (to appear).

[31] A. Lerner, A "local mean oscillation" decomposition and some its applications, Function spaces, Approximation, Inequalities and Lineability, Lectures of the Spring School in Analysis, Matfyzpress, Prague (2011), 71-106.

[32] S. Lu, Y. Ding AND D. YAN, Singular integrals and related topics, World Scientific, (2007).

[33] M. Mastylo And Y. Sawano, Complex interpolation and Calderón-Mityagin couples of Morrey spaces, Anal. PDE 12, (2019) 1711-1740.

[34] M. Mastylo, Y. Sawano And H. TanaKa, Morrey type space and its Köthe dual space, Bull. Malays. Math. Soc., 41, (2018) 1181-1198.

[35] C. Morrey, On the solution of quasi-linear elliptic partial differential equations, Trans. Am. Math. Soc. 43 (1938), 126-166.

[36] E. NAKAI, Hardy-Littlewood maximal operator, singular integral operators and the Riesz potentials on generalized Morrey spaces, Math. Nachr. 166 (1994), 95-103.

[37] E. NAKAI, A characterization of pointwise multipliers on the Morrey spaces, Sci. Math. 3, (2000) 445-454.

[38] S. Nakamura, T. Noi And Y. Sawano, Generalized Morrey spaces and trace operator, Sci. China Math., 59, (2016) 281-336.

[39] S. Nakamura, Y. Sawano and H. Tanaka, Weighted local Morrey spaces, Ann. Acad. Sci. Fenn. Math. 45 (2020), 67-93.

[40] A. NeKVINDA, Hardy-Littlewood maximal operator on $L^{p(x)}\left(\mathbb{R}^{n}\right)$, Math. Inequal. Appl., 7 (2004), $255-265$.

[41] M. Ramseyer, O. Salinas And B. Viviani, Two-weighted norm inequalities for the local maximal function, J. Geom. Anal. 27 (2017), 120-141.

[42] J. Rubio De Francia, Factorization and extrapolation of weights, Bull. Amer. Math. Soc. (N. S.), 7 (1982), 393-395. 
[43] J. Rubio DE Francia, A new technique in the theory of $A_{p}$ weights, Topics in modern harmonic analysis, Vol. I, II (Turin/Milan, 1982), Ist. Naz. Alta Mat. Francesco Severi, Rome, 1983, 571-579.

[44] J. Rubio de Francia, Factorization theory and $A_{p}$ weights, Amer. J. Math., 106 (1984), 533-547.

[45] Y. SAWANo, A vector-valued sharp maximal inequality on Morrey spaces with non-doubling measures, Georgian Math. J., 13, (2006) 153-172.

[46] Y. SAWANO, S. SUGANO AND H. TANAKA, Generalized fractional integral operators and fractional maximal operators in the framework of Morrey spaces, Trans. Amer. Math. Soc., 363 (2011) 64816503.

[47] Y. Sawano, A thought on generalized Morrey spaces, J. Indones. Math. Soc. 25, (2019), $210-281$.

[48] Y. SAwANo, K.-P. Ho, D. YAng And S. YAnG, Hardy spaces for ball quasi-Banach function spaces, Dissertationes Mathematicae, 525 (2017), 1-102.

[49] Y. SaWAno AND H. Yoshida, A predual of a predual of $B_{\sigma}$ and its applications to commutators, Sci. China Math. 61, (2018), 1437-1472.

[50] X. SHI, Two inequalities related to geometric mean operators, J. Zhejiang Teacher's College 1 (1980), $21-25$.

[51] J.-O. S trömberG, Bounded mean oscillation with Orlicz norms and duality of Hardy spaces, Indiana Univ. Math. J. 28 (1979), 511-544. 